\title{
Expert Group Consensus Opinion on Prostate Cancer Diagnosis and Management in India: Part 1 of 2
}

\author{
Amit Ghose ${ }^{1}$, Makarand Khochikar ${ }^{2}$, Ravindra Sabnis ${ }^{3}$, \\ Naveen Magendra Parmar ${ }^{4}$, Indranil Purkait ${ }^{4}$ \\ ${ }^{1}$ Apollo Gleneagles Hospitals Limited, Kolkata, India \\ ${ }^{2}$ Department of Uro-Oncology, Siddhi Vinayak Ganapati Cancer Hospital, Miraj, India \\ ${ }^{3}$ Department of Urology, Muljibhai Patel Urological Hospital, Nadiad, Gujarat, India \\ ${ }^{4}$ Medical Affairs and Clinical Research, Ipca Laboratories Ltd., Mumbai, India
}

Purpose: To evaluate Indian literature, clinical practice and develop consensus opinion on various aspects of prostate cancer diagnosis and management in order to assist medical practitioners across India in effectively choosing right treatment option for patients with prostate cancer.

Materials and Methods: At the CAP summit held in Kolkata on 2019, a group of leading experts from across India voted on 42 consensus statements. Final statements were derived on the basis of voting results and subsequent expert panel meeting. Based on the panellists discussion on various areas of prostate cancer and practicability of recommendation in clinical practice, 5 statements were deleted and a draft was prepared. The draft is divided into 2 parts; part 1 covers 16 statements on prostate cancer diagnosis and early prostate cancer. Results: A total of 37 statements were accepted and finalised by expert panel members based on the voting results. Sixteen statements with varying degrees of support from the panel are described in detail in this article. Conclusions: The statements derived from several Indian scientific evidences, local clinical experience, and international guidelines will serve as a reference guide for clinicians across India in the management of prostate cancer. (Korean J Urol Oncol 2020;18:170-182)

Key Words: Prostate cancer $\cdot$ Guideline $\cdot$ Prostate-specific antigen $\cdot$ Prostatectomy consensus

\section{INTRODUCTION}

Prostate cancer $(\mathrm{PCa})$ is predominantly a disease of elderly and more than 3 quarter of cases occurs in men above the age of 65 years. ${ }^{1}$ It is the second most common cancer

Received July 24, 2020, Revised August 10, 2020,

Accepted September 11, 2020

Corresponding Author: Naveen Magendra Parmar

Medical Affairs and Clinical Research, Ipca Laboratories Ltd., Ipca Laboratories, 142-B, Charkop Rd, Charkop Industrial Estate, Charkop,

Kandivali West, Mumbai 400067, Maharashtra, India

E-mail: naveenparmar95@gmail.com

Tel: +91-9867423441

ORCID: https://orcid.org/0000-0002-5452-7006 and the sixth leading cause of cancer death amongst men worldwide. ${ }^{2}$ The data on true incidence of PCa in India is limited as this entity is not a notifiable disease and there are very few population-based cancer registries. ${ }^{1}$ PCa had the fifth highest incidence rate among males in India in 2016 (4.8 per 100,000; 95\% uncertainty interval [UI], 3.8$5.8)$, with $33,000(26,000-40,000)$ incident cases and 112,000 $(87,000-137,000)$ prevalent cases. The age-standardized incidence rate of $\mathrm{PCa}$ increased significantly by $29.8 \%(95 \%$ UI, 8.5-46.9) from 1990 to $2016 .^{3}$ The 5-year survival rate of patients with $\mathrm{PCa}$ is much lower in India (58.1\%) as compared to Western nations $(>90 \%)$ with higher incidence. ${ }^{4}$ Also, compared to the Western countries it was

This is an Open Access article distributed under the terms of the Creative Commons Attribution Non-Commercial License (http://creativecommons.org/licenses/by-nc/4.0/) which permits unrestricted non-commercial use, distribution, and reproduction in any medium, provided the original work is properly cited. 2020 (C) Copyright The Korean Urological Oncology Society and The Korean Prostate Society. All Rights Reserved. 
thought that the prevalence of PCa in India is lower but with the increased migration of rural population to the urban areas, increased awareness, changing life style and easy access to medical facility, more cases of PCa are being picked up and this suggests that India is not very far behind from Western countries in the rate of $\mathrm{PCa}$. The cancer projection data reveals that the number of cases will double by the year $2020 .^{2}$

Hence, it is important to have consensus on the issue of $\mathrm{PCa}$ - early diagnosis by using latest medical diagnostic tests. The prostate-specific membrane antigen positron emission tomography (PSMA-PET) scan is now rapidly being incorporated to help in staging of the disease. Also, much has been achieved for curing early $\mathrm{PCa}$ using radical robotic prostatectomy and radical radiotherapy. Consensus on various issues related to $\mathrm{PCa}$ would enable cure and achieve a better quality of care in patients. Also, huge advances have been made in chemotherapy, hormone therapy, and salvage radiotherapy for advanced disease. This would control advanced PCa and provide relief. Consensus on advanced PCa management is also required in order to get optimum control and a better quality of life.

\section{MATERIALS AND METHODS}

Experts in the field of urology from all over India were invited to CAP summit and a 2-day consultative meeting was convened under the aegis of Board of Education, Urological Society of India on 24th-25th of May, 2019 at Kolkata, India. As a part of the consensus process (Fig. 1),
4 major areas were defined for discussion, namely prostate cancer diagnosis, early prostate cancer, locally advanced prostate cancer, and metastatic prostate cancer. In each of the sections, the issues were determined according to perceived clinical importance. A total of 42 consensus statements were designed and voting of 28 experts was taken with the help of voting pads. Each major area was discussed on various international guidelines and evidences followed by voting on consensus statements. The grade of the evidence and the level of agreement were based on voting results (Table 1). When the proportion of those who voted was $80 \%$ or higher, the statement was regarded as strongly accepted. The result of the consensus and manuscript version one was discussed with the expert panel of 13 members (Table 2) in the consensus board meeting on 29th November 2019 before drafting the final manuscript draft. The meeting resulted in deletion of 5 statements according to the practicability of recommendation. This manuscript is the outcome of the expert group discussion and consensus comprising 16 statements on prostate cancer diagnosis and early prostate cancer.

Table 1. Grading system for consensus statement

\begin{tabular}{ll}
\hline \multicolumn{1}{c}{ Voting percentage } & Recommendation strength \\
\hline$>80 \%$ & Strong \\
$60 \%-80 \%$ & Moderate \\
$50 \%-60 \%$ & Weak \\
\hline
\end{tabular}

\begin{tabular}{|c|}
\hline Identification of most important areas in prostate cancer diagnosis and management \\
\hline Panel selection (28 members) \\
\hline Draft consensus questions \\
\hline Consensus questions are debated and voted during Kolkata conference 24-25 May 2019 \\
\hline Collation of responses and determining grading criteria \\
\hline Draft/manuscript v1 formulated \\
\hline Consensus board meeting held on 29 November 2019 \\
\hline Final draft/manuscript circulated amongst members of consensus board meeting \\
\hline Post approval manuscript submission to peer-reviewed journal for publication \\
\hline
\end{tabular}

Fig. 1. Consensus process flow. 


\section{RESULTS}

\section{Prostate Cancer Diagnosis}

1) Statement No.1.

Is there a role of digital rectal examination (DRE) in prostate cancer diagnosis?

Table 2. Consensus board meeting members and their speciality

\begin{tabular}{ll}
\hline \multicolumn{1}{c}{ Name } & Speciality \\
\hline Dr. Amit Ghose & Urologist \\
Dr. Ajay Kumar & Urologist \\
Dr. Anant Kumar & Urologist \\
Dr. Aneesh Srivastava & Urologist \\
Dr. C Mallikarjuna & Urologist \\
Dr. Makarand Khochikar & Urologist \\
Dr. N P Gupta & Urologist \\
Dr. Prem Kumar & Urologist \\
Dr. Rajeev Kumar & Urologist \\
Dr. Ravindra Sabnis & Urologist \\
Dr. S K Raghunath & Urologist \\
Dr. S K Singh & Urologist \\
Dr. Sudhir Rawal & Urologist
\end{tabular}

Literature review: In a case series published by Ghagane et al., ${ }^{5} 87.5 \%$ of 471 PCa cases had abnormal DRE and a significant association was observed between DRE and prostate-specific antigen (PSA) level $(p=0.0005){ }^{5}$ In the study of Jariwala, ${ }^{6}$ PSA was not available in 40 cases and PCa was found in 14 cases on prostate biopsy, 35\% being positive only on abnormal DRE. In another study, at multiple PSA ranges, suspicious DRE finding significantly increased the cancer detection rates as compared to nonsuspicious DRE. ${ }^{4}$ Table 3 shows positive predictive value (PPV) of PSA and DRE in 876 patients who underwent biopsy. ${ }^{7}$ In a retrospective study of 922 patients, DRE examination revealed abnormalities in 158 subjects $(17.1 \%)$. The relationship between the number of subjects with abnormal DRE findings and their PSA levels is shown in Table 4; as the PSA levels increased the number of prostate cancer cases detected by DRE also increased. ${ }^{8}$

Consensus: Is there a role of DRE in prostate cancer diagnosis? (yes: $85.7 \%$, no: $3.5 \%$, no response: $10.7 \%$ )

\section{2) Statement No.2.}

Is there a role for prostate cancer screening in India?

Literature review: There are no randomized control trials

Table 3. Positive predictive values (PPV) of prostate-specific antigen (PSA) and digital rectal examination (DRE) in patients who underwent prostate biopsy

\begin{tabular}{|c|c|c|c|c|}
\hline PSA range $(\mathrm{ng} / \mathrm{mL})$ & DRE & Biopsy done & Cancer & PPV (\%) \\
\hline \multirow[t]{2}{*}{$<4$} & Negative & 0 & - & - \\
\hline & Positive & 25 & 5 & 20.0 \\
\hline \multirow[t]{2}{*}{$4.1-10$} & Negative & 216 & 33 & 15.2 \\
\hline & Positive & 47 & 28 & 59.57 \\
\hline \multirow[t]{2}{*}{$10.1-20$} & Negative & 96 & 23 & 24.0 \\
\hline & Positive & 60 & 41 & 68.3 \\
\hline \multirow[t]{2}{*}{$>20$} & Negative & 115 & 72 & 62.60 \\
\hline & Positive & 316 & 301 & 95.2 \\
\hline Total & & 875 & 503 & \\
\hline
\end{tabular}

Table 4. Digital rectal examination findings at varying prostate-specific antigen (PSA) levels

\begin{tabular}{lcc}
\hline \multicolumn{1}{c}{ PSA $(\mathrm{ng} / \mathrm{mL})$} & No. of subjects with abnormalities on DRE & Detection rate of prostate cancer \\
\hline$<4(\mathrm{n}=490)$ & $14(2.86)$ & $3(0.61)$ \\
$4-10(\mathrm{n}=171)$ & $20(11.7)$ & $4(2.34)$ \\
$10-20(\mathrm{n}=118)$ & $15(12.71)$ & $3(2.54)$ \\
$20-50(\mathrm{n}=41)$ & $35(85.37)$ & $14(34.15)$ \\
$>50(\mathrm{n}=102)$ & $74(72.55)$ & $56(54.9)$ \\
\hline
\end{tabular}

Values are presented as number $(\%)$. 
on population-based PCa screening in India. However, in a study conducted by Agnihotri et al., ${ }^{7}$ of the 4,702 patients, $70.9 \%$ had PSA level $<4 \mathrm{ng} / \mathrm{mL}$ and $29.1 \%$ had PSA level $>4 \mathrm{ng} / \mathrm{mL}$. Eleven point eight percent had between 4.1-10 $\mathrm{ng} / \mathrm{mL}, 5.4 \%$ men had PSA level between $10.1-20 \mathrm{ng} / \mathrm{mL}$, and $11.8 \%$ had $>20 \mathrm{ng} / \mathrm{mL}$. PSA test positivity rate was $29.1 \%$. In this study, although there was a higher PSA test positivity, the PPV of PSA test in symptomatic men was low. This suggests that an increased number of PSA positive men are unnecessarily put through biopsy. ${ }^{7}$ It is now accepted that PSA screening prevents approximately one PCa death per 1,000 men, each screened several times and followed for 10-15 years. ${ }^{9}$ Overdiagnosis of prostate cancer (estimated to be 35 overdiagnosed cases per 1,000 men screened), a large majority of which are low-risk cancers that would never progress or metastasize, is the most important harm that outweighs the benefits of screening. ${ }^{9}$ Dubey $^{10}$ in his review analysed evidences on the usefulness of PSA screening and concluded that there is no scientific rationale to advocate routine use of PSA for early detection of PCa in Indian males. The Government policy in favour of screening for prostate cancer does not exist in India and thus practising population-based screening is difficult.

Consensus: Is there a role for prostate cancer screening in India? (yes: $32.1 \%$, no: $67.9 \%$, no response: $0 \%$ )

\section{3) Statement No. 3}

Should all men have a multiparametric magnetic resonance imaging (mp-MRI) of the prostate/pelvis prior to making a decision to proceed to biopsy?

Literature review: In one prospective study conducted in India, the diagnostic performance of mp-MRI was analysed in a group of subjects with serum PSA of $\leq 10 \mathrm{ng} / \mathrm{mL}$. Receiver operating characteristic (ROC) analysis of Prostate
Imaging Reporting and Data System (PIRADS-S) score showed area under the curve of $0.93(p<0.001)$ for the detection of PCa. Youden selected threshold cutoff was 8 ( $\geq$ 8 ) with sensitivity, specificity, PPV, and negative predictive value (NPV) of $85 \%, 87.88 \%, 68 \%$, and $95.1 \%$, respectively. Mp-MRI showed high NPV with PIRADS-S score cutoff, ${ }^{8}$ which suggest the ability of the test to predict the absence of disease with high confidence. This factor can be used to limit unnecessary biopsy of prostate. ${ }^{11}$ In another study conducted in 26 patients, sensitivity, specificity, PPV, and NPV of T2, diffusion-weighted imaging, and magnetic resonance spectroscopy (MRS) in predicting malignancy was calculated after which transrectal ultrasonography (TRUS) guided biopsy was performed; results of which are described in Table 5. ${ }^{12}$ Prebiopsy mp-MRI should become standard of care as recommended in 2019 NICE guidelines [NG131]. However, its implementation has proved to be difficult in the United Kingdom and certainly in India.

Consensus: Should all men have a mp-MRI of the prostate/pelvis prior to making a decision to proceed to biopsy? (yes: $25 \%$, no: $67.8 \%$, no response: $7.1 \%$ )

\section{4) Statement No. 4}

Should transrectal ultrasound-guided biopsies remain the standard approach?

Literature review: Gupta et al. ${ }^{13}$ reported a false negative rate of approximately $11 \%$ with TRUS-guided sextant biopsy. In symptomatic men with negative DRE, Agnihotri et al. ${ }^{7}$ proposed raising the serum PSA cutoff to $5.4 \mathrm{ng} / \mathrm{mL}$ for TRUS biopsy in India as this would prevent $10 \%$ unnecessary biopsies. This reflects low $\mathrm{PCa}$ detection rates of TRUS biopsy in India. It has been observed that compared to other continents, Asian population (including India) have a lower TRUS biopsy yield, especially for serum PSA level

Table 5. Sensitivity, specificity, positive predictive value (PPV), and negative predictive value (NPV) of T2, DWI, and MRS in predicting malignancy

\begin{tabular}{lllll}
\hline \multicolumn{1}{c}{ Variable } & T2W & MRS & DWI & Combined (T2+DWI+MRS) \\
\hline Sensitivity & $94.7 \%$ & $84.2 \%$ & $89.5 \%$ & $94.7 \%$ \\
Specificity & $42.9 \%$ & $28.6 \%$ & $85.7 \%$ & $42.9 \%$ \\
PPV & $81.8 \%$ & $76.2 \%$ & $74.4 \%$ & $81.8 \%$ \\
NPV & $75 \%$ & $40 \%$ & $75 \%$ & $75 \%$ \\
Diagnostic accuracy & $80.8 \%$ & $69.2 \%$ & $88.5 \%$ & $80.8 \%$ \\
\hline
\end{tabular}

DWI: diffusion-weighted imaging, MRS: magnetic resonance spectroscopy, T2W: T2-weighted. 
$<20 \mathrm{ng} / \mathrm{mL}$. Patil et al., ${ }^{4}$ have confirmed these findings and thus standard serum PSA cutoff of $4 \mathrm{ng} / \mathrm{mL}$ followed in Western nations gives lower TRUS biopsy yield for Indian population. Cancer detection rates of TRUS biopsies from Indian studies are shown in Table 6.

Consensus: Should transrectal ultrasound-guided biopsies remain the standard approach? (yes: $85.7 \%$, no: $14.3 \%$, no response: $0 \%$ )

\section{5) Statement No. 5}

What imaging modalities should be used to complement the mp-MRI of the pelvis/prostate when determining the presence/absence of metastatic disease?

Literature review: There are no Indian studies on direct comparison of PSMA-PET-computed tomography (CT) and Isotope bone scan-CT. In the study of Gupta et al., ${ }^{14}$ in their experience of 97 staging prostate cancer patients, PSMAPET-CT showed $57.41 \%$ with only sclerotic metastasis. $33.33 \%$ mixed, $7.14 \%$ marrow, and $2.3 \%$ lytic types of lesions constitute the rest; therefore, bone scan alone in these patients may result in underestimation of bony disease burden. ${ }^{14}$ In another study, ${ }^{68}$ Ga-PSMA PET CT and MRI diagnostic sensitivity, specificity, PPV, NPV, and accuracy for detection of lymph node metastasis in high-risk cases were $66.67 \%, 98.61 \%, 85.71 \%, 95.95 \%, 95.06 \%$, and $25.93 \%, 98.61 \%, 70 \%, 91.42 \%, 90.53 \%$, respectively. ${ }^{15}$ In a retrospective study of 262 patients having PCa or suspected recurrent $\mathrm{PCa},{ }^{68} \mathrm{Ga}-\mathrm{PSMA}$ scan detected extraprostatic disease in $53.2 \%$ of subjects when done at baseline prior to starting any treatment. In this study, the sensitivity of ${ }^{68} \mathrm{Ga}$-PSMA at baseline with histopathological diagnosis was $95 \%$ and the PPV was $98 \%{ }^{16}$ PSMA-PET-CT is a suitable replacement for conventional imaging as it provides su- perior accuracy than CT scan and bone scan.

Consensus: What imaging modalities should be used to complement the mp-MRI of the pelvis/prostate when determining the presence/absence of metastatic disease? (PSMAPET-CT: $57.1 \%$, isotope bone scan+CT scan: $39.2 \%$, no response: $3.57 \%$ )

\section{6) Statement No. 6}

Should all prostate cancer biopsies be reviewed by a pathologist with specific experience in the interpretation of prostate cancer histology?

Literature review: In the study of Singh et al., ${ }^{17}$ general pathologists scored $\mathrm{PCa}$ cases using Gleason grade. For Gleason score (GS) groups $(2-4,5-6,7$, and 8-10) overall agreement with consensus score was $68 \%$. For primary grade $60 \%$ of readings were in fair to moderate agreement range; for secondary grade $78 \%$ of readings were in slight to fair agreement range. For GS, $80 \%$ of readings were in slight to fair agreement range; and for GS groups $68.5 \%$ of the readings were in fair to moderate agreement range. In practice, it has been seen that general pathologists often underscore than overscore. In the same study, overscoring was observed in $33.7 \%$ and underscoring in $23 \%{ }^{17}$ In another study, after attending a web-based tutorial, pathologist scored PCa cases independently using the Gleason scoring system. Pathologist overgraded $15 \%$ cases of score 7 and $12.5 \%$ cases of scores 5-6 in the pretutorial round. In posttutorial round, this score was reduced to $11.25 \%$ and 10.6 , respectively. ${ }^{18}$

Consensus: Should all prostate cancer biopsies be reviewed by a pathologist with specific experience in the interpretation of prostate cancer histology? (yes: $89.2 \%$, no: $10.7 \%$, no response: $0 \%$ )

Table 6. Comparison with cancer detection rates of transrectal ultrasonography biopsy reported in India

\begin{tabular}{|c|c|c|c|c|c|c|c|c|}
\hline \multirow{2}{*}{ Study } & \multirow{2}{*}{$\begin{array}{l}\text { No. of } \\
\text { patients }\end{array}$} & \multicolumn{7}{|c|}{ Cancer detection rate $(\%)$} \\
\hline & & Overall & $\mathrm{PSA}<4$ & PSA $4-10$ & PSA $10-20$ & PSA $20-50$ & PSA $50-100$ & $\mathrm{PSA}>100$ \\
\hline \multirow[t]{2}{*}{ Agnihotri et al. ${ }^{7}$} & 875 & 57.5 & $20^{\dagger}$ & $15.2 *$ & $24^{*}$ & $62.6^{*}$ & & \\
\hline & & & & $59.57^{\dagger}$ & $68.3^{\dagger}$ & $95.2^{\dagger}$ & & \\
\hline Sinha et al. ${ }^{40}$ & 119 & 24.37 & - & 7.14 & 6.67 & 52.17 & & \\
\hline Patil et al. $^{4}$ & 235 & 25.53 & - & 5.95 & 13.16 & 32.26 & 100 & 100 \\
\hline
\end{tabular}

PSA: prostate-specific antigen, DRE: digital rectal examination.

*Normal DRE. ${ }^{\dagger}$ Abnormal DRE. 


\begin{tabular}{|c|c|c|}
\hline $\mathrm{S} / \mathrm{N}$ & Statement & Strength of recommendation \\
\hline 1 & $\begin{array}{l}\text { Is there a role of DRE in prostate cancer diagnosis? } \\
\text { Yes: } 85.7 \% \text {, no: } 3.5 \% \text {, no response: } 10.7 \% \\
\text { There is a role of DRE in prostate cancer diagnosis }\end{array}$ & Strong \\
\hline 2 & $\begin{array}{l}\text { Is there a role for prostate cancer screening in India? } \\
\text { Yes: } 32.1 \% \text {, no: } 67.9 \% \text {, no response: } 0 \% \\
\text { There is no role for prostate cancer screening in India }\end{array}$ & Moderate \\
\hline 3 & $\begin{array}{l}\text { Should all men have a mp-MRI of the prostate/pelvis prior to making a decision to } \\
\text { proceed to biopsy? } \\
\text { Yes: } 25 \% \text {, no: } 67.8 \% \text {, no response: } 7.1 \% \\
\text { All men should not have a mp-MRI of the prostate/pelvis prior to making a } \\
\text { decision to proceed to biopsy }\end{array}$ & Moderate \\
\hline 4 & $\begin{array}{l}\text { Should transrectal ultrasound-guided biopsies remain the standard approach? } \\
\text { Yes: } 85.7 \% \text {, no: } 14.3 \% \text {, no response: } 0 \% \\
\text { Transrectal ultrasound-guided biopsies should remain the standard approach }\end{array}$ & Strong \\
\hline 5 & $\begin{array}{l}\text { What imaging modalities should be used to complement the mp-MRI of the } \\
\text { pelvis/prostate when determining the presence/absence of metastatic disease? } \\
\text { PSMA-PET-CT: } 57.1 \% \text {, isotope bone scan+CT scan: } 39.2 \% \text {, no response: } 0 \% \\
\text { PSMA-PET-CT should be used to complement the mp-MRI of the pelvis/prostate } \\
\text { when determining the presence /absence of metastatic disease }\end{array}$ & Weak \\
\hline 6 & $\begin{array}{l}\text { Should all prostate cancer biopsies be reviewed by a pathologist with specific } \\
\text { experience in the interpretation of prostate cancer histology? } \\
\text { Yes: } 89.2 \% \text {, no: } 10.7 \% \text {, no response: } 0 \% \\
\text { All prostate cancer biopsies should be reviewed by a pathologist with specific } \\
\text { experience in the interpretation of prostate cancer histology }\end{array}$ & Strong \\
\hline
\end{tabular}

\section{Early Prostate Cancer}

\section{1) Statement No. 1}

Is active surveillance (AS) a viable management option for low-risk prostate cancer in the Indian context?

Literature review: There are no Indian guidelines or studies on the role of AS in prostate cancer. Data of AS in more than 4,000 PCa patients with median follow-up of 4.5 years has been shown in Table $7 .^{19}$ In Indian setting, patients generally have insufficient medical knowledge and their treatment outcome expectation is high. In addition, the large Indian population is not regular for AS follow-up. Medical violence is also quite common, thus it is difficult for urologists in India to face the medical disputes caused by $\mathrm{PCa}$ progression during AS. Implementing AS in India is therefore difficult. Even conditions in China are similar to India, which is why radical prostatectomy is still the primary choice of Chinese urologist in low-risk prostate cancer, followed by $\mathrm{AS}^{20}$

Consensus: Is AS a viable management option for low-risk prostate cancer in the Indian context? (yes: 42.9\%, no: $57.1 \%$, no response: $0 \%$ )

\section{2) Statement No. 2}

Should Gleason Grade Grouping (GG) be adopted across India as a standard for reporting histological grade?

Literature review: In a retrospective study comparing the GS of 2005 and 2015 criteria, there was a marked decrease $(80 \%)$ in GS 6; among these cases, $80 \%$ cases were graded as score 7 , and $20 \%$ cases were graded as score 8 . There was also a $28.57 \%$ decrease in GS 8 and $60 \%$ increase in GS 9 due to the new criteria for pattern 4 . The GG 1, 2, 3 , 4, and 5 constituted $3.03 \%, 18.18 \%, 15.15 \%, 15.15 \%$, and $48.49 \%$ of cases respectively. ${ }^{21}$ In another Indian study, applying World Health Organization 2016 modified Gleason scoring system and prognostic grade grouping criteria to PCa biopsies, there was upgradation of prognostic groups in $7.69 \%$ patients. There was also an increase in GS $4+3$ from $19.6 \%$ to $23.8 \%$ and a decrease in GS $3+4$ from $13.9 \%$ to $9 \%$; After follow-up of 2 years poor prognoses was observed in those patients who were upgraded to the higher prognostic group. ${ }^{22}$ 
Table 7. Studies of active surveillance in prostate cancer

\begin{tabular}{lrccc}
\hline \multicolumn{1}{c}{ Study } & Patients (n) & Median follow-up (yr) & Freedom from treatment & Prostate cancer death \\
\hline PRIAS 2013 & & & & \\
Selvadurai et al. & 2,494 & 1.6 & $77 \%$ at $2 \mathrm{yr}$ & 0 \\
Tosoian et al. $^{43}$ & 471 & 5.7 & $70 \%$ at $5 \mathrm{yr}$ & $0.40 \%$ \\
Dall'Era et al. $^{44}$ & 769 & 2.7 & $59 \%$ at $5 \mathrm{yr}$ & 0 \\
Thomsen et al. $^{45}$ & 321 & 3.6 & $67 \%$ at $5 \mathrm{yr}$ & 0 \\
SAMS $^{*} 2013^{46}$ & 167 & 3.4 & $\mathrm{NA}$ & $\mathrm{NA}$ \\
Klotz et al. $^{47}$ & 148 & $\mathrm{NA}$ & $70 \%$ at $5 \mathrm{yr}$ & $3 \%$ at $15 \mathrm{yr}$ \\
Total & 450 & 6.8 & & \\
\hline
\end{tabular}

NA: not applicable, PRIAS: Prostate Cancer Research International Active Surveillance, SAMS: Study of Active Monitoring in Sweden.

*Prospective and randomized.

Consensus: Should Gleason Grade Grouping be adopted across India as a standard for reporting histological grade? (yes: $100 \%$, no: $0 \%$, no response: $0 \%$ )

\section{3) Statement No. 3}

Does the management and prognosis for high-risk localized and locally advanced prostate cancer differ?

Literature review: The current standard of care in management of high-risk and locally advanced disease is external beam radiotherapy (EBRT) in combination with longterm androgen deprivation therapy (ADT); specifically, a 3-dimensional conformal radiation therapy or intensity-modulated radiation therapy (IMRT) radiation therapy technique with a dose of 75-80 Gy in conjunction with long-term ADT in a neoadjuvant, concurrent, or adjuvant setting for 2-3 years. In general, brachytherapy is not considered for treatment in high-risk patients; however, in certain scenario brachytherapy boost could be used in conjunction with EBRT, along with consideration of short-term ADT. In addition, surgery could be considered in selected high-risk patients, even though, it is not a popular approach because of it is invasive in nature compared to EBRT. Also, surgery has it's complication which is long-term sexual dysfunction, urinary incontinence etc. It's highly likely that postoperative radiotherapy would be required which will expose patients to toxicities of radiotherapy as well as surgery. ${ }^{23}$

Consensus: Does the management and prognosis for highrisk localized and locally advanced prostate cancer differ? (yes: $75 \%$, no: $17.8 \%$, no response: $7.1 \%$ )

\section{4) Statement No. 4}

Should hypo fractionated radiotherapy (60 Gy in 20 fractions) be considered the standard radiotherapy regimen for low-to-intermediate risk prostate cancer?

Literature review: In an Indian study, patients with organconfined PCa received hypofractionated IMRT. Dose prescribed to the prostate was $77 \mathrm{~Gy} / 35$ fractions or $60 \mathrm{~Gy} / 20$ fractions. After a median follow-up of 26 months, there were 6 biochemical relapses, 1 in intermediate and 5 in high-risk group, of whom 3 deaths occurred due to distant metastasis. In this study, the 2-year biochemical recurrencefree survival (bRFS), prostate cancer-specific survival (pCSS), and overall survival (OS) were 92.3\%, 93.9\%, and 91.5\% respectively. Twenty percent and $13.3 \%$ developed acute and late Radiation Therapy Oncology Group (RTOG) grade 2 or worse gastrointestinal (GI) toxicities respectively. Eight point nine percent and $11.1 \%$ developed RTOG grade 2 or worse genitourinary (GU) toxicity respectively. One patient developed grade 3 rectal toxicity, and none developed bladder toxicity. ${ }^{24}$ In another Indian study, no significant difference was observed in the incidence of grade 2 GI and GU toxicities between the 2 schedules of $60 \mathrm{~Gy}$ in 20 fractions and 65 Gy in 25 fractions. ${ }^{25}$

Consensus: Should hypo fractionated radiotherapy (60 Gy in 20 fractions) be considered the standard radiotherapy regimen for low-to-intermediate risk prostate cancer? (yes: $57.1 \%$, no: $35.7 \%$, no response: $7.1 \%$ )

\section{5) Statement No. 5}

Should radical prostatectomy be considered as a management option for men with high-risk (D'Amico) $\mathrm{cN} 0 \mathrm{cM} 0$ 
prostate cancer?

Literature review: Most patients in India present with locally advanced disease or metastatic disease due to lack of medical education and awareness. Even those patients presenting with localized disease who could be managed with $\mathrm{RP}$, are in the high-risk group. In an Indian study, patients underwent robotic-assisted radical prostatectomy (RARP) and it was observed that $39.6 \%$ were in high-risk group. After a follow-up of 12 months post-RARP, 27.8\% had biochemical recurrence (BCR), and 92\% patients were continent. ${ }^{26}$ Kulkarni et al. ${ }^{27}$ studied the outcome of upfront RP in Indian patients with high risk PCa. From 1996 to 2010, 208 PCa patients (high-risk category D'Amico's criteria) underwent open RP with bilateral pelvic lymphadenectomy. At 7 and 10 years, pCSS was found to be $79.7 \%$ and $65 \%$, respectively, bRFS was $42.4 \%$ and $36.7 \%$, respectively and the metastasis-free survival (MFS) was $71.1 \%$ and $64.4 \%$ respectively. ${ }^{27}$ In another study, out of 192 patients who underwent RP 109 had D'Amico high-risk (HR) disease. The 2- and 5-year bRFSs were $45 \%$ and $35 \%$, respectively. It was also observed that the 2 -year bRFS was $63 \%, 23 \%$, and $22 \%$, for $1 \mathrm{HR}, 2 \mathrm{HR}$, and $3 \mathrm{HR}$ respectively (log rank, $\mathrm{p}<0.0001){ }^{28}$ In the study of Mishra et al., ${ }^{29}$ after undergoing laparoscopic radical prostatectomy for a clinical T2 localized disease, patient's 5-year progression-free probability with low-, intermediate-, and high-risk PCa was 91\%, $82 \%$, and $58 \%$, respectively. ${ }^{29}$

Consensus: Should radical prostatectomy be considered as a management option for men with high-risk (D'Amico) cN0 cM0 prostate cancer? (yes: 85.7 , no: $7.1 \%$, no response: $7.1 \%$ )

\section{6) Statement No. 6}

Should lymph node dissection be recommended in all men with high-risk (D’Amico) $\mathrm{cN} 0 \mathrm{cM} 0$ prostate cancer undergoing a radical prostatectomy?

Literature review: Lymph node positivity is detected in $5 \%-6 \%, 20 \%-25 \%$, and $30 \%-40 \%$ of low-, intermediate-, and high-risk $\mathrm{PCa}$ patients respectively who undergo RP and extended pelvic lymph node dissection (ePLND). ${ }^{30}$ In contrast to the Western countries, most Indian patients are detected with higher stage of PCa and with greater probability of lymph node involvement. Results of the study of Batra et al. ${ }^{31}$ showed that $7 \%$ and $45 \%$ of patients had low-risk and high-risk disease respectively and positive lymph node was found in $20 \%$ of these high-risk patients on ePLND. This demonstrates how much important pelvic lymphadenectomy is in patients undergoing RARP in the Indian subcontinent. Abdollah et al. ${ }^{32}$ studied the practice patterns in the utilization of pelvic lymph node dissection in Indian and United States (US) practices. Even this study confirmed that Indian patients had a higher risk distribution compared to US $(53.4 \%$ in India vs. $27 \%$ in the US; $\mathrm{p}<0.001$ ). It was also observed that Indian patients with low risk more frequently underwent PLND (81.0\% vs. 41.4\%). In addition, the study showed that the probability of Indian patients undergoing PLND was 2.57-fold higher than their US counterparts. ${ }^{32}$

Consensus: Should lymph node dissection be recommended in all men with high-risk (D'Amico) cN0 cM0 prostate cancer undergoing a radical prostatectomy? (yes: 92.8, no: $0 \%$, no response: $7.1 \%$ )

\section{7) Statement No. 7}

In men undergoing lymph node dissection during radical prostatectomy for high-risk (D'Amico) cN0 cM0 prostate cancer, which lymph node regions should be dissected?

Literature review: With the absence of Indian literature, evidences from international studies have been evaluated. In high-risk men with lymph node positive disease after RP and ePLND, Bader et al. ${ }^{33}$ found that the common sites for metastasis in patients were obturator fossa $(60 \%)$ followed by internal iliac (hypogastric) (58\%) and external iliac nodal areas $(36 \%)$. Nineteen percent of the patients had metastasis in the hypogastric region alone. In another study, 470 lymph nodes were detected scintigraphically and $46 \%$ positive nodes were found in patients during ePLND. If ePLND went only up to the common iliac chain, 94\% would have been correctly staged, but only $77 \%$ would have had all their metastasised sites removed. ${ }^{30}$ A recent study of PLND versus ePLND evaluated bPFS outcomes in localized PCa patients undergoing open RP. The 5-year bPFS in low risk, intermediate-risk, and high-risk patients undergoing PLND and ePLND were found out to be $90.1 \%$ and $91.3 \%, 73.1 \%$ and $85.7 \%, 51.1 \%$, and $71.4 \%$ respectively. ${ }^{34}$

Consensus: In men undergoing lymph node dissection during radical prostatectomy for high-risk (D'Amico) $\mathrm{cN} 0$ cM0 prostate cancer, which lymph node regions should be 
dissected? (below common illiac bifurcation: $64.2 \%$, above common iliac bifurcation: $32.1 \%$, no response: $3.5 \%$ ). In the discussion during Consensus Board Meeting (CBM), expert committee was of the opinion that the statement derived from this question should have a strong recommendation.

\section{8) Statement No. 8}

Which staging investigations should be performed in a man with high-risk (D'Amico) cN0 cM0 prostate cancer prior to confirming suitability for surgery?

Literature review: There are no Indian studies on comparison of various investigations in high-risk (D'Amico) $\mathrm{cN} 0$ cM0 prostate cancer prior to confirming suitability for surgery. However, Indian data on different investigations in prostate cancer are mentioned in statement No. 5 under the diagnosis section of consensus opinion. Due to lack of Indian data, evidences from other literature were assessed. In a study conducted in Jordan, ${ }^{68}$ Ga PSMA PET/CT scan in high-risk disease patients showed a higher concordance rate of $90 \%$ as compared to the bone scan $(75 \%)$ followed by MRI scan (73\%), and CT scan $(60 \%)$. The study also demonstrated that ${ }^{68} \mathrm{Ga}$ PSMA PET/CT was more accurate in detecting suspicious pelvic lymph nodes than MRI scan (95.2\% vs. $80 \%$ ) but it had almost same accuracy to MRI in detecting PCa lesions. In this study ${ }^{68} \mathrm{Ga}$ PSMA PET/CT also performed better than CT scan in identifying suspicious lymph nodes $(95.2 \%$ vs. $75 \%)$ and extrapelvic nodes $(100 \%$ vs. $75 \%)$, as well as bone lesions via bone scan (100\% vs. $62.5 \%$ ). Intriguingly the management of $\mathrm{PCa}$ was changed by ${ }^{68} \mathrm{Ga}$ PSMA PET/CT in $52 \%$ patients. ${ }^{35}$

Consensus: Which staging investigations should be performed in a man with high-risk (D'Amico) $\mathrm{cN} 0 \mathrm{cM} 0$ prostate cancer prior to confirming suitability for surgery? (multiparameteric MRI+bone scan: 35.7\%, PSMA-PET: $60.7 \%$, no response: $3.5 \%$ )

\section{9) Statement No. 9}

Should neoadjuvant ADT be considered for men with high-risk (D’Amico) cN0 cM0 prostate cancer prior to radical prostatectomy?

Literature review: Indian studies are lacking on this subject, thus other available literature was reviewed for this statement. Narita et al. ${ }^{36}$ studied the outcomes in patients having high-risk disease treated with neoadjuvant chemo- hormonal therapy (NCHT) with RP alone. In this study, $10 \%$ patients had pathologic complete response and 3.3\% had positive surgical margins. The BCR-free survival rates for 2 years and 5 years were $69.2 \%$ and $60.1 \%$, respectively. After propensity score matching, it was observed that the $\mathrm{BCR}$ rate in patients remained significantly lower in the NCHT+RP group than RP group alone. ${ }^{36}$ In 2006 a Cochrane review published that neoadjuvant ADT improved pathological outcomes but not overall or disease-free survival. ${ }^{37}$

Consensus: Should neoadjuvant ADT be considered for men with high-risk (D'Amico) cN0 cM0 prostate cancer prior to radical prostatectomy? (yes: $28.5 \%$, no: $64.2 \%$, no response: $7.1 \%$ )

\section{0) Statement No. 10}

Is there a role for trimodality therapy (ADT+radical prostatectomy+adjuvant radiotherapy [ART]) for men with high-risk (D’Amico) cN0 cM0 prostate cancer?

Literature review: Trials have demonstrated that combination therapies improve survival and cancer outcomes when compared to monotherapy for men with high-risk PCa. To date, only one prospective randomized trial comparing RP plus ADT with EBRT plus ADT in the treatment of patients with high-risk $\mathrm{PCa}$ has been published; men with $\mathrm{T}^{-} \mathrm{b}^{-}$ 3N0M0 tumours were included in this trial, regardless of serum PSA level. After a follow-up of 102 months, the 10 -year OS rate in the patients who underwent surgery tended to be better than the rate observed in the radiation group (76.2\% vs. $71.1 \%)$. Furthermore, other outcomes were improved in the surgery cohort compared to radiotherapy cohort: bPFS was $83.5 \%$ versus $66.1 \%$, clinical progressionfree survival rates were $85.7 \%$ versus $77.1 \%$, and diseasespecific survival (DSS) rates was $67.9 \%$ versus $60.9 \%$. However, none of these survival advantages reached statistical significance. ${ }^{38}$ In another retrospective study, the outcome in PCa patients receiving combination of ART and ADT after RP was evaluated. After a median follow-up of 61 months, the 5- and 7-year bRFS rates were 90.5 and $77.2 \%$, respectively. Distant relapse occurred in 5 patients, resulting in 5- and 7-year MFS of $95.9 \%$ and $81.7 \%$, respectively. During follow-up, 7 patients died (2 PCa deaths), resulting in 5- and 7-year DSS and OS rates of $100 \%$ and $94.7 \%$ and 90.6 and $81.5 \%$, respectively. ${ }^{39}$

Consensus: Is there a role for trimodality therapy 


\begin{tabular}{|c|c|c|}
\hline $\mathrm{S} / \mathrm{N}$ & Statement & Strength of recommendation \\
\hline 1 & $\begin{array}{l}\text { Is AS a viable management option for low-risk prostate cancer in the Indian context? } \\
\text { Yes: } 42.9 \% \text {, no: } 57.1 \% \text {, no response: } 0 \% \\
\text { AS is not a viable management option for low-risk prostate cancer in the Indian context }\end{array}$ & Weak \\
\hline 2 & $\begin{array}{l}\text { Should Gleason Grade Grouping be adopted across India as a standard for reporting } \\
\text { histological grade? } \\
\text { Yes: } 100 \% \text {, no: } 0 \% \text {, no response: } 0 \% \\
\text { Gleason Grade Grouping should be adopted across India as a standard for reporting } \\
\text { histological grade }\end{array}$ & Strong \\
\hline 3 & $\begin{array}{l}\text { Does the management and prognosis for high-risk localized and locally advanced } \\
\text { prostate cancer differ? } \\
\text { Yes: } 75 \% \text {, no: } 17.8 \% \text {, no response: } 7.1 \% \\
\text { The management and prognosis for high-risk localized and locally advanced prostate } \\
\text { cancer should differ }\end{array}$ & Moderate \\
\hline 4 & $\begin{array}{l}\text { Should hypo fractionated radiotherapy ( } 60 \text { Gy in } 20 \text { fractions) be considered the } \\
\text { standard radiotherapy regimen for low-to-intermediate risk prostate cancer? } \\
\text { Yes: } 57.1 \% \text {, no: } 35.7 \% \text {, no response: } 7.1 \%\end{array}$ & Weak \\
\hline ss & $\begin{array}{l}\text { Hypo fractionated radiotherapy ( } 60 \mathrm{~Gy} \text { in } 20 \text { fractions) should be considered the } \\
\text { standard radiotherapy regimen for low-to-intermediate risk prostate cancer }\end{array}$ & \\
\hline 5 & $\begin{array}{l}\text { Should radical prostatectomy be considered as a management option for men with } \\
\text { high-risk (D'Amico) cN0 cM0 prostate cancer? } \\
\text { Yes: } 85.7 \% \text {, no: } 7.1 \% \text {, no response: } 7.1 \% \\
\text { Radical prostatectomy should be considered as a management option for men with } \\
\text { high-risk (D'Amico) cN0 cM0 prostate cancer }\end{array}$ & Strong \\
\hline 6 & $\begin{array}{l}\text { Should lymph node dissection be recommended in all men with high-risk (D'Amico) } \\
\text { cN0 cM0 prostate cancer undergoing a radical prostatectomy? } \\
\text { Yes: } 92.8 \% \text {, no: } 0 \% \text {, no response: } 7.1 \% \\
\text { Lymph node dissection should be recommended in all men with high-risk (D'Amico) } \\
\text { cN0 cM0 prostate cancer undergoing a radical prostatectomy }\end{array}$ & Strong \\
\hline 7 & $\begin{array}{l}\text { In men undergoing lymph node dissection during radical prostatectomy for high-risk } \\
\text { (D'Amico) cN0 cM0 prostate cancer, which lymph node regions should be dissected? } \\
\text { Below common iliac bifurcation: } 64.2 \% \text {, above common iliac bifurcation: } 32.1 \% \text {, no } \\
\text { response: } 3.5 \% \\
\text { In men undergoing lymph node dissection during radical prostatectomy for high-risk } \\
\text { (D'Amico) cN0 cM0 prostate cancer the lymph node regions below common iliac } \\
\text { bifurcations should be dissected }\end{array}$ & Moderate \\
\hline 8 & $\begin{array}{l}\text { Which staging investigations should be performed in a man with high-risk (D'Amico) } \\
\text { cN0 cM0 prostate cancer prior to confirming suitability for surgery? } \\
\text { Multiparameteric MRI+bone scan: } 35.7 \% \text {, PSMA-PET: } 60.7 \% \text {, no response: } 3.5 \% \\
\text { PSMA-PET should be performed in a man with high-risk (D'Amico) cN0 cM0 prostate } \\
\text { cancer prior to confirming suitability for surgery }\end{array}$ & Moderate \\
\hline 9 & $\begin{array}{l}\text { Should neoadjuvant ADT be considered for men with high-risk (D'Amico) cN0 cM0 } \\
\text { prostate cancer prior to radical prostatectomy? } \\
\text { Yes: } 28.5 \% \text {, no: } 64.2 \% \text {, no response: } 7.1 \% \\
\text { Neoadjuvant ADT should not be considered for men with high-risk (D'Amico) cN0 } \\
\text { cM0 prostate cancer prior to radical prostatectomy }\end{array}$ & Moderate \\
\hline 10 & $\begin{array}{l}\text { Is there a role for trimodality therapy (ADT+radical prostatectomy+ART) for men with } \\
\text { high-risk (D'Amico) cN0 cM0 prostate cancer? } \\
\text { Yes: } 53.5 \% \text {, no: } 42.8 \% \text {, no response: } 3.5 \% \\
\text { There is a role for trimodality therapy (ADT+radical prostatectomy+ART) for men with } \\
\text { high-risk (D'Amico) cN0 cM0 prostate cancer }\end{array}$ & Weak \\
\hline
\end{tabular}


$(\mathrm{ADT}+$ radical prostatectomy+ART) for men with high-risk (D'Amico) cN0 cM0 prostate cancer? (yes: 53.5\%, no: 42.8\%, no response: $3.5 \%$ ). During CBM, the expert committee was of the opinion that the statement derived from this question should have a strong recommendation and the terminology trimodality is to be replaced with multimodality.

\section{CONFLICT OF INTEREST}

The authors claim no conflicts of interest.

\section{ACKNOWLEDGMENTS}

We gratefully acknowledge the contribution of all the participants who voted in the CAP summit on prostate cancer consensus statements. We are thankful to all the expert panel members of the consensus board meeting for their lively and stimulating discussion on the statements. We thank Dr. Prokar Dasgupta for his guidance and help in conducting the CAP summit. We thank Dr. Simon Hughes for his contribution in designing the consensus questions, conducting background research work, and reviewing the manuscript. We also thank Dr. Ashutosh Tewari for his active participation in clinical discussions during the CAP summit.

\section{REFERENCES}

1. Hariharan K, Padmanabha V. Demography and disease characteristics of prostate cancer in India. Indian J Urol 2016;32:103-8.

2. Jain S, Saxena S, Kumar A. Epidemiology of prostate cancer in India. Meta Gene 2014;2:596-605.

3. India State-Level Disease Burden Initiative Cancer Collaborators. The burden of cancers and their variations across the states of India: the Global Burden of Disease Study 1990-2016. Lancet Oncol 2018;19:1289-306.

4. Patil SR, Pawar PW, Sawant AS, Patil AV, Narwade SS, Mundhe ST, et al. TRUS biopsy yield in Indian population: a retrospective analysis.. J Clin Diagn Res 2017; 11:PC01-5.

5. Ghagane SC, Nerli RB, Hiremath MB, Wagh AT, Magdum PV. Incidence of prostate cancer at a single tertiary care center in North Karnataka. Indian J Cancer 2016; 53:429-31.

6. Jariwala SK. Prostate biopsy in a rural hospital: our experience. BLDE Univ J Health Sci 2017;2:18-21.

7. Agnihotri S, Mittal RD, Kapoor R, Mandhani A. Raising cut-off value of prostate specific antigen (PSA) for biopsy in symptomatic men in India to reduce unnecessary biopsy. Indian J Med Res 2014;139:851-6.

8. Chavan PR, Chavan SV, Chavan NR, Trivedi VD. Detection rate of prostate cancer using prostate specific antigen in patients presenting with lower urinary tract symptoms: a retrospective study. J Postgrad Med 2009;55: 17-21.

9. Mandal R, Basu P. Cancer screening and early diagnosis in low and middle income countries: current situation and future perspectives. 2018;61:1505-12.

10. Dubey D. The routine use of prostate-specific antigen for early detection of cancer prostate in India: is it justified? Indian J Urol 2009;25:177-84.

11. Koshy SM, Prahladan A, Kalidos K, Ramachandran KN. Role of multiparametric MRI prostate as a screening tool for cancer detection. JMSCR 2017;5:18162-76.

12. Jagannathan D, Indiran V. Accuracy of diffusion weighted images and MR spectroscopy in prostate lesions - our experience with Endorectal coil on 1.5 T MRI. J Clin Diagn Res 2017;11:TC10-4.

13. Gupta NP, Ansari MS, Dass SC. Transrectal ultrasound guided biopsy for detecting early prostate cancer: an Indian experience. Indian J Cancer 2005;42:151-4.

14. Gupta M, Choudhury PS, Goel HC, Rawal S, Talwar V. Is PSMA PET-CT better than bone scan? When and why. J Nucl Med Radiat Ther 2017;8:100342.

15. Gupta M, Choudhury PS, Hazarika D, Rawal S. A comparative study of 68Gallium-prostate specific membrane antigen positron emission tomography-computed tomography and magnetic resonance imaging for lymph node staging in high risk prostate cancer patients: an initial experience. World J Nucl Med 2017;16:186-91.

16. Kallur KG, Ramachandra PG, Rajkumar K, Swamy SS, Desai I, Rao RM, et al. Clinical utility of gallium-68 PSMA PET/CT scan for prostate cancer. Indian J Nucl Med 2017;32:110-7.

17. Singh RV, Agashe SR, Gosavi AV, Sulhyan KR. Interobserver reproducibility of Gleason grading of prostatic adenocarcinoma among general pathologists. Indian J Cancer 2011;48:488-95.

18. Mulay K, Swain M, Jaiman S, Gowrishankar S. Gleason scoring of prostatic carcinoma: impact of a web-based tutorial on inter- and intra-observer variability. Indian $\mathrm{J}$ Pathol Microbiol 2008;51:22-5.

19. Lund L, Svolgaard N, Poulsen MH. Prostate cancer: a review of active surveillance. Res Rep Urol 2014;6:107-12.

20. Wei Y, Liu L, Li X, Song W, Zhong D, Cao X, et al. Current treatment for low-risk prostate cancer in china: a national network survey. J Cancer 2019;10:1496-502. 
21. Shah MB, Raju K, Kumar GH. Revisiting prostate biopsy with 2014 ISUP modified Gleason score and Gleason grade - a cross section study. Biomed Res Ther 2018;5: 2918-25.

22. Rao V, Garudadri G, Shilpa AS, Fonseca D, Sudha SM, Sharma R, et al. Validation of the WHO 2016 new Gleason score of prostatic carcinoma. Urol Ann 2018;10: 324-9.

23. Marciscano AE, Hardee ME, Sanfilippo N. Management of high-risk localized prostate cancer. Adv Urol 2012; 2012:641689.

24. Goswami J, Mallik S, Mondal M, Sheet S, Das S, Sen A, et al. Preliminary results of clinical outcomes with hypofractionated intensity modulated radiotherapy in organ confined prostate cancer: an Indian experience. Cancer Ther Oncol Int J 2018;11:555823.

25. Arunsingh M, Mallick I, Prasath S, Arun B, Sarkar S, Shrimali RK, et al. Acute toxicity and its dosimetric correlates for high-risk prostate cancer treated with moderately hypofractionated radiotherapy. Med Dosim 2017;42:18-23.

26. Gupta NP, Murugesan A, Kumar A, Yadav R. Analysis of outcome following robotic assisted radical prostatectomy for patients with high risk prostate cancer as per D'Amico classification. Indian J Urol 2016;32:115-9.

27. Kulkarni JN, Gunavanthe VS, Dhale A. Outcome of radical prostatectomy as primary treatment for high-risk prostate cancer patients. Indian J Cancer 2015;52:646-52.

28. Bijalwan P, Pooleri GK, Kalavampara SV, Bhat S, Thomas A, Sundar P, et al. Pathological outcomes and biochemical recurrence-free survival after radical prostatectomy for high-risk prostate cancer in the Indian population. Indian J Urol 2018;34:260-7.

29. Mishra S, Agrawal V, Khatri N, Sharma R, Kurien A, Ganpule A, et al. Laparoscopic radical prostatectomy: oncological outcome analysis from a single-center Indian experience of 6 years. Indian J Urol 2012;28:32-6.

30. Chopra S, Alemozaffar M, Gill I, Aron M. Extended lymph node dissection in robotic radical prostatectomy: Current status. Indian J Urol 2016;32:109-14.

31. Batra V, Gautam G, Jaipuria J, Suryavanshi M, Khera R, Ahlawat R. Predictive factors for lymph node positivity in patients undergoing extended pelvic lymphadenectomy during robot assisted radical prostatectomy. Indian J Urol 2015;31:217-22.

32. Abdollah F, Arora S, Jindal T, Gild P, Sood A, Yuvaraja $\mathrm{TB}$, et al. Utilization of pelvic lymph node dissection in patients undergoing robot-assisted radical prostatectomy in India versus the United States - A Vattikuti Collective Quality Initiative database analysis. Indian J Cancer 2017; 54:421-5.

33. Bader P, Burkhard FC, Markwalder R, Studer UE. Is a limited lymph node dissection an adequate staging proce- dure for prostate cancer? J Urol 2002;168:514-8.

34. Ji J, Yuan H, Wang L, Hou J. Is the impact of the extent of lymphadenectomy in radical prostatectomy related to the disease risk? A single center prospective study. J Surg Res 2012;178:779-84.

35. Hirmas N, Al-Ibraheem A, Herrmann K, Alsharif A, Muhsin H, Khader J, et al. [68Ga]PSMA PET/CT improves initial staging and management plan of patients with high-risk prostate cancer. Mol Imaging Biol 2019;21: 574-81.

36. Narita S, Nara T, Kanda S, Numakura K, Saito M, Inoue $\mathrm{T}$, et al. Radical prostatectomy with and without neoadjuvant chemohormonal pretreatment for high-risk localized prostate cancer: a comparative propensity score matched analysis. Clin Genitourin Cancer 2019;17:e11322.

37. Kumar S, Shelley M, Harrison C, Coles B, Wilt TJ, Mason MD. Neo-adjuvant and adjuvant hormone therapy for localised and locally advanced prostate cancer. Cochrane Database Syst Rev 2006;(4):CD006019.

38. Akakura K, Suzuki H, Ichikawa T, Fujimoto H, Maeda O, Usami $\mathrm{M}$, et al. A randomized trial comparing radical prostatectomy plus endocrine therapy versus external beam radiotherapy plus endocrine therapy for locally advanced prostate cancer: results at median follow-up of 102 months. Jpn J Clin Oncol 2006;36:789-93.

39. Omrcen T, Hrepic D, Boraska Jelavic T, Vrdoljak E. Combination of adjuvant radiotherapy and androgen deprivation therapy after radical prostatectomy in high risk prostate cancer patients - results from retrospective analysis. J BUON 2015;20:1061-7.

40. Sinha S, Siriguri SR, Kanakmedala SK, Bikkasani K. Prostate biopsy findings in Indian men: a hospital-based study. Indian J Cancer 2011;48:175-80.

41. Bul M, Zhu X, Rannikko A, Staerman F, Valdagni R, Pickles $\mathrm{T}$, et al. Radical prostatectomy for low-risk prostate cancer following initial active surveillance: results from a prospective observational study. Eur Urol 2012;62: 195-200.

42. Selvadurai ED, Singhera M, Thomas K, Mohammed K, Woode-Amissah R, Horwich A, et al. Medium-term outcomes of active surveillance for localised prostate cancer. Eur Urol 2013;64:981-7.

43. Tosoian JJ, Trock BJ, Landis P, Feng Z, Epstein JI, Partin AW, et al. Active surveillance program for prostate cancer: an update of the Johns Hopkins experience. J Clin Oncol 2011;29:2185-90.

44. Dall'Era MA, Albertsen PC, Bangma C, Carroll PR, Carter $\mathrm{HB}$, Cooperberg MR, et al. Active surveillance for prostate cancer: a systematic review of the literature. Eur Urol 2012;62:976-83.

45. Thomsen FB, Brasso K, Klotz LH, Røder MA, Berg KD, 
Iversen P. Active surveillance for clinically localized prostate cancer - a systematic review. J Surg Oncol 2014;109: $830-5$.

46. Bratt Ol, Carlsson S, Holmberg E, Holmberg L, Johansson E, Josefsson A, et al. The Study of Active Monitoring in Sweden (SAMS): a randomized study comparing two dif- ferent follow-up schedules for active surveillance of low-risk prostate cancer. Scand J Urol 2013;47:347-55.

47. Klotz L, Zhang L, Lam A, Nam R, Mamedov A, Loblaw A. Clinical results of long-term follow-up of a large, active surveillance cohort with localized prostate cancer. J Clin Oncol 2010;28:126-31. 